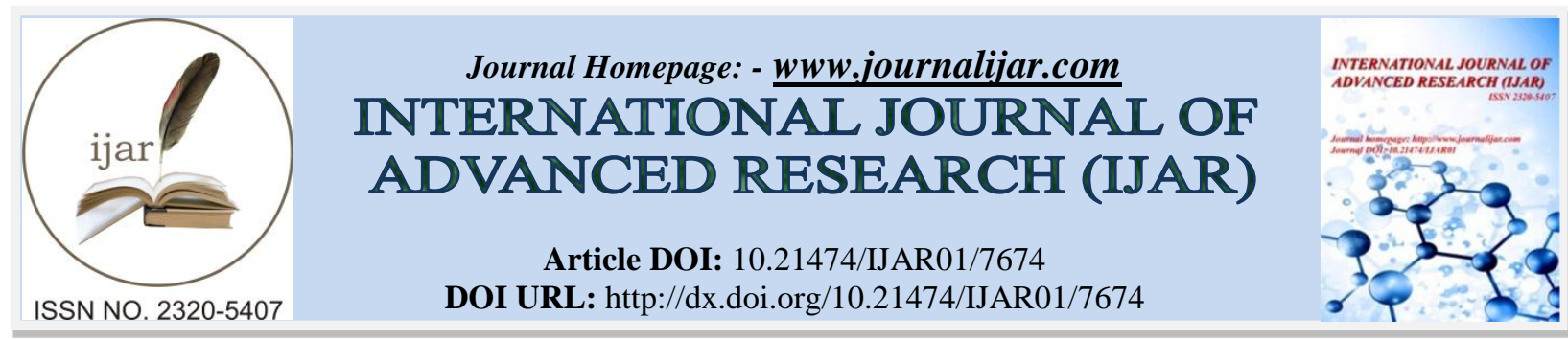

RESEARCH ARTICLE

\title{
IDENTIFICATION OF THE STRATA OF LATERITIC SOILS AND ALTERITES IN BENIN.
}

\author{
Victor S. Gbaguidi ${ }^{1}$, Yvette Tankpinou Kiki ${ }^{2}$, Crépin Zevounou ${ }^{1}$, Nestor Vedogbeton ${ }^{1}$ and Marcel Zankpe ${ }^{1}$. \\ 1. University of Abomey-Calavi (UAC). \\ 2. National University of Sciences, Technologies, Engineering and Mathematics (UNSTIM).
}

\section{Manuscript Info}

Manuscript History

Received: 05 July 2018

Final Accepted: 07 August 2018

Published: September 2018

Keywords:-

lateritic soils, alterites, reserves.

\begin{abstract}
This paper aims at exploring the sites of raw materials used in the production of laterite briquettes in Benin Republic. Specifically, the work was based on the investigation, delimitation and evaluation of the reserves of lateritic soils and alterites that may be included in the production of laterite briquettes.

The tests showed thirty-three (33) favorable sites among which eighteen (18) lateritic soils and fifteen (15) alterites identified in the twelve Benin departments on the plateaux of the coastal sedimentary basin (lateritic soils) as well as on the crystalline bedrock, the Buem and the sedimentary basin (alterite) of Kandi. The various sections of wells obtained revealed a homogeneity on the sites of lateritic soils. Moreover, they appear as coherent soils while alterites present a more or less indurate appearance.

The bedrock profiles varied between 1.2 and $2.2 \mathrm{~m}$ comparatively to plateaux where the depths of the wells reached more than $5 \mathrm{~m}$. The established reserves are estimated to be $10410949 \mathrm{~m}^{3}$ for lateritic soils and $724806 \mathrm{~m}^{3}$ for alterites; which represents a great asset with regard to the high price of usual construction materials and the environmental challenge. Thus, local materials impose themselves day by day.
\end{abstract}

Copy Right, IJAR, 2018,. All rights reserved.

\section{Introduction:-}

Given the dual reality of standard building materials high cost and the environmental challenge, the use of local building materials is becoming an obvious solution. Also, the prohibition of extraction of the sea sand and the improvement living conditions of the population regarding dwelling have made the promotion of local building materials in Benin (Vedogbeton and Olodo, 1996). As such emphasis has been placed on the production and use of self-stabilized soil agglomerates from Lateritic soils and alterite available on Benin coastal sedimentary basin and bedrock (Igué and al., 2005 and 2012; Houessou and Lang, 1978).Those agglomerates are already used in some areas and would allow building ecological, bio-climatic constructions (Sorgbo, 2016). In order to achieve this promotion, the study aims at searching and characterizing the local building materials under consideration, namely lateritic soils and alterites, used in the manufacture of agglomerates. This will help making up the lack of technical studies on raw materials and self-blocking agglomerates.

Lateritic soils represents the most dominant soils in southern Benin; they are ironed soils, weakly desaturated sandyclay on clayey sediments of Continental Terminal or Cretaceous sandstone (Slansky, 1962).The upper part of the 
Continental Terminal being recently described by the Breda Research Institute as Upper Miocene and PliocenePleistocene (IRB, 1985). Alterites are primeval cover formations associated with the weathering and evolution of climatic and pedogenetic superficial formations in place (crystalline bedrock) or weathered (alluvium, alluvial manuring); they result from the very extreme alteration of the source rock, which is no longer recognizable (Igué and al., 2012).

The goal of this research aims at exploring, delimitating and evaluating the reserve sites where stabilized, selfblocking soil agglomerates raw material (lateritic soils, alterites) are available enough and economically exploitable, according to environmental standards.

\section{Materials and Methods:-}

Site searching

Benin is a West African country, covering an area of $114.764 \mathrm{~km}^{2}$ and stretching from $6^{\circ}$ to $12{ }^{\circ} \mathrm{N}$ latitude over 700 $\mathrm{km}$ with an annual rainfall of 900 to $1400 \mathrm{~mm}$. It is subdivided into seventy-seven (77) communes distributed in twelve (12) departments (Atacora, Atlantique, Borgou, Mono, Oueme, Zou, Alibori, Collines, Couffo, Donga, Littoral and Plateau).

The country has five main geological sites:1) the coastal sedimentary basin; 2) the Paleo-Mesozoic basin of Kandi; 3) the outer area of the Dahomeyides Pan-African chain, including the tectonic units of Atacora (quartzites, shales, prasinites, sandstones), Buem (sandstone, jasper, volcano-sediments), and their foreland, the Pendjari basin (mudstones, siltstones, clay and sandstones); 4) the internal area of the pan-African chain of Dahomeyides, including the Benin plain structural unit commonly called crystalline bedrock and pan-African syn- to- tectonic intrusions; 5) volcano-sedimentary formations consisting of rhyolites, basalts and dolerites associated with molasses' series.

Lateritic soils used in the production of stabilized, self-blocking agglomerates, is the upper part of Benin southern plateaux. These are Come, Allada and Sakete plateaux in the south of the Lama depression and those of Aplahoue, Abomey, Ketou and the small Zagnanado plateau in the North of the depression (Fig 1).

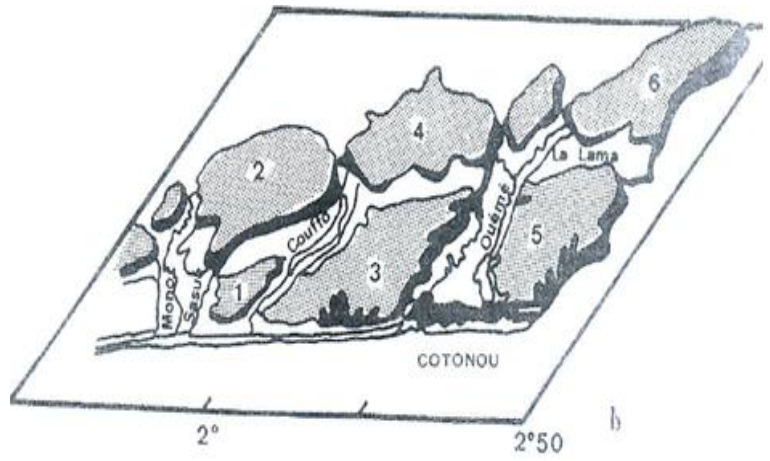

Figure 1:-Distribution of geographical units in Southern Benin: 1. Come Plateau; 2. Aplahoue Plateau; 3. Allada Plateau; 4. Abomey Plateau; 5. Sakete Plateau; 6. Ketou Plateau (Houessou et Lang, 1978)

In the central communes (Dassa-Zoume, Savalou, Glazoue and Save) where the Precambrian crystalline basement is mainly made up of gneissic, migmatic, pan-African granite formations as well as volcano-sedimentary formations of Daho-Mahou (sandstones, conglomerates, rhyolites, andesites, basalts, volcanic breccias ...) (IRB, 1985). In the North, the basement is essentially made up of migmatites, granulites, meta-sediments, high degree of metamorphism gneisses, part of the Benin Plain Structural Unit and pan-African syn-to-tectonic intrusions represented by porphyroid granites, finely to moderately granular, gabbro and monzo-syenites (Affaton, 1973; OBEMINES, 1984 ; 1989-a ; 1989-b ; 1995).

\section{Calculation of reserves}

In the field, the coordinates of the summits of each site have been recorded. At the end of the works, the perimeters of the prospected sites were determined and their areas as well. Knowing the thickness of the raw material based on 
the wells' depth, the reserves were calculated in cubic meters $\left(\mathrm{m}^{3}\right)$ then in tonne $(\mathrm{t})$. These reserves are proven reserves, given the spatial continuity and depth of the prospected layers.

\section{Sampling}

Compared to potential sites, especially those selected for sinking wells, three (03) research wells were dug, and each of them, down to a depth of $5 \mathrm{~m}$ for each plateau. The well has a square section, hand dug with $1 \mathrm{~m}$ as side. Wells are sampled from $1 \mathrm{~m}$ passes piles. The constitution of the sample, obtained representing the entire well by quartering. To obtain a sample, equal proportions are taken from each pile after quartering (Fig 2).

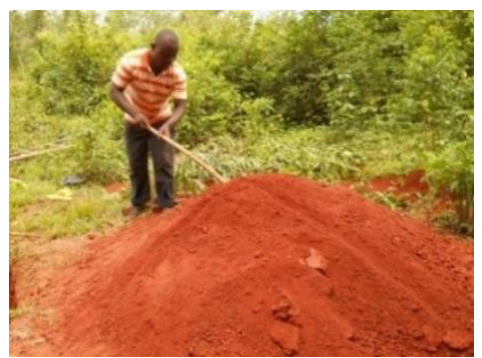

Figure 2:-Quartering on a $1 \mathrm{~m}$ pass

\section{Findings and Discussions:-}

The investigations identified eighteen (18) sites at the level of the coastal sedimentary basin and fifteen (15) sites at the basement level in large agglomerations, taking into account geological features.

For the lateritic soils of the seven trays, the communes explored are: Abomey, Abomey-Calavi, Adja-Ouere, Adjarra, Agbangnizoun, Allada, Aplahoue, Bohicon, Bopa, Come, Dogbo, Houeyogbe, Ketou, Lokossa, Ouidah, Sakété, Zagnanado and Za-Kpota (Fig 3). For the alterites, the work took place (Fig 3) in the communes of the Center (Dassa-Zoumè, Glazoue, Ouesse, Savalou, and Save) and the North (Banikoara, Bassila, Djougou, Kandi, Kouande, Natitingou, Nikki, Parakou, Tanguiéta and Tchaourou). 


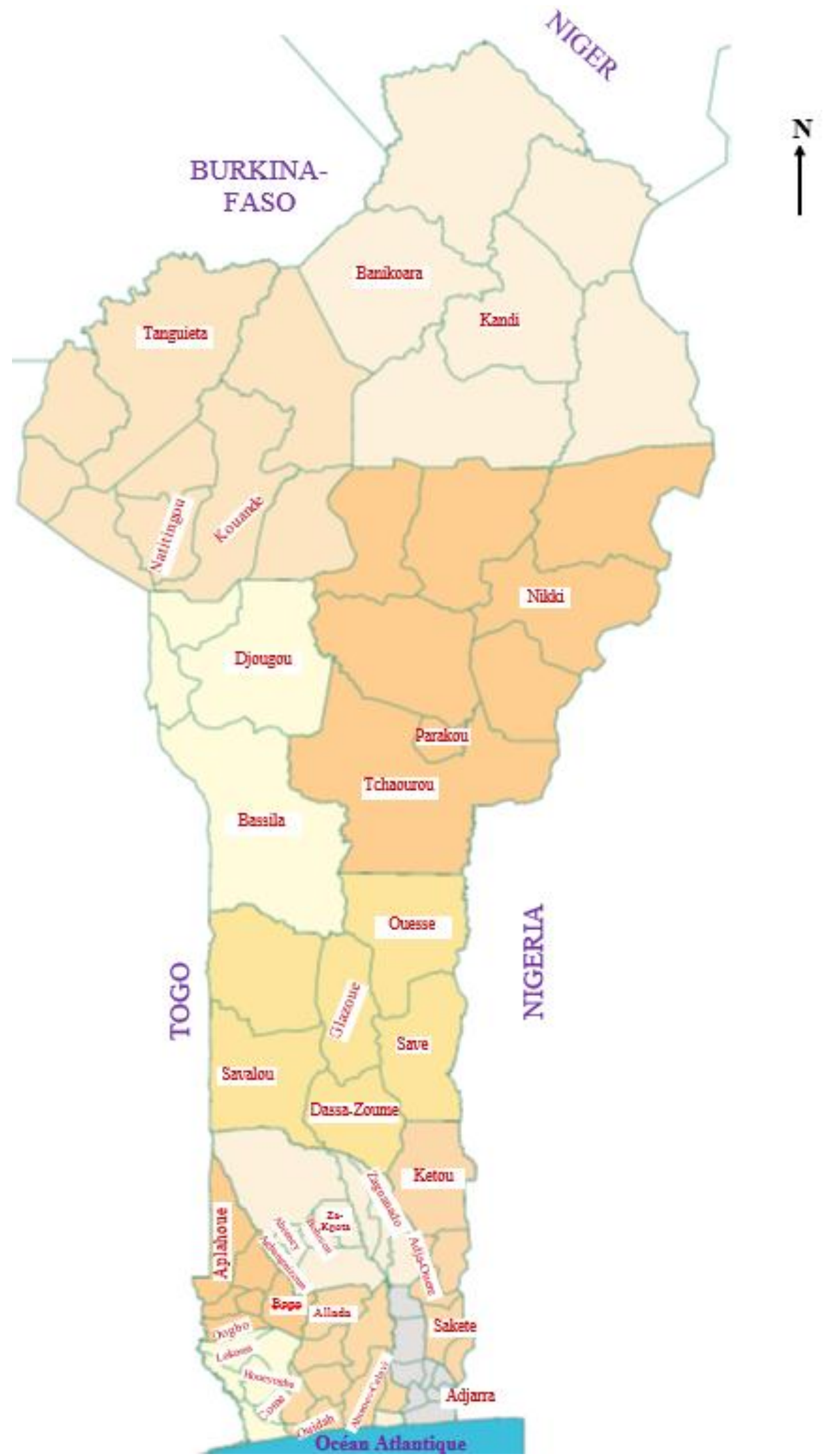

Figure 3:-Investigated sites locations on Benin map 


\section{Features}

Figure 4 and Figure 5 present some images taken during the implantation, excavation and closing of wells.

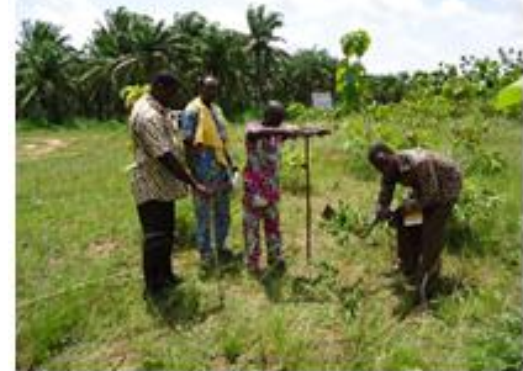

(a) Implantation of a wells

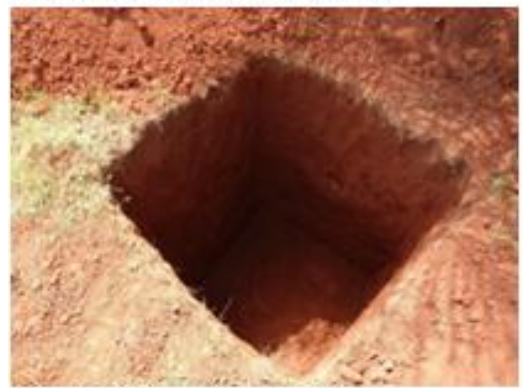

(c) Well dug in Ouidah

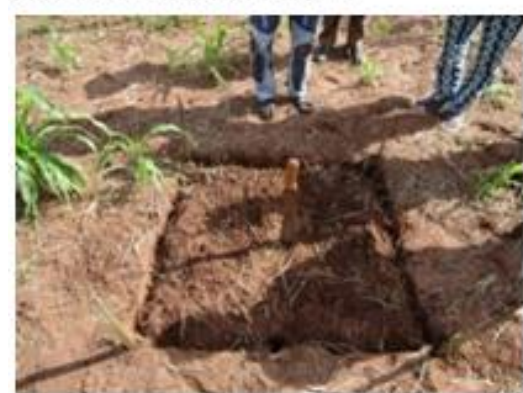

(e) Well dug in Aplahoue

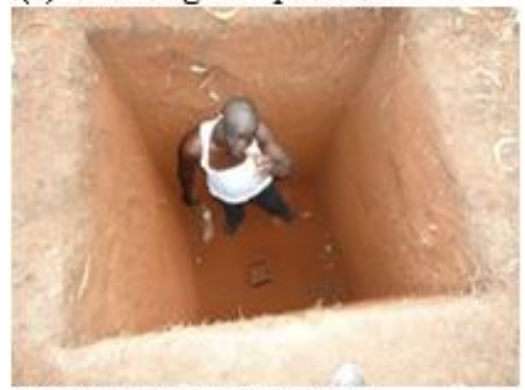

(g) Well dug in Abomey

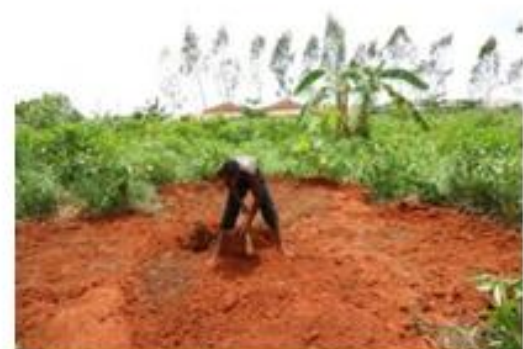

(b) Closing the well dug in Allada

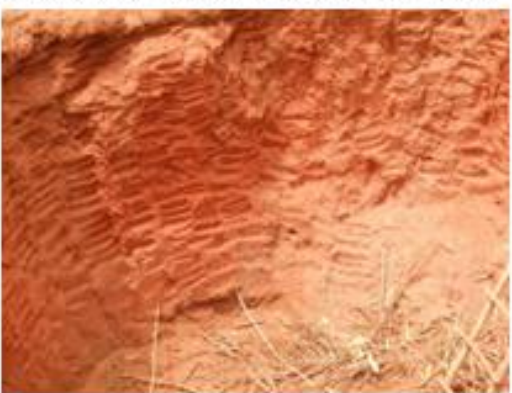

(d) Well dug in Adjarra

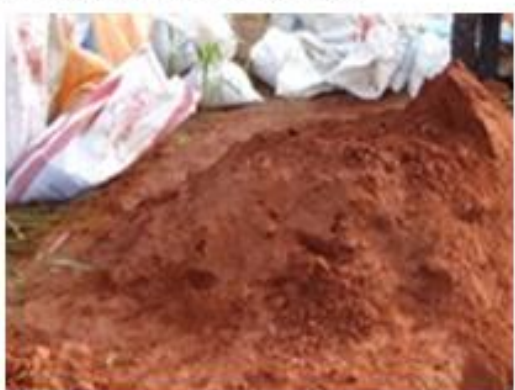

(f) Modified sample in Lokossa

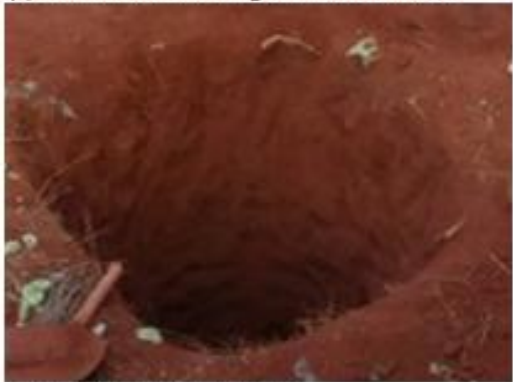

(h) Well dug in Ketou

Figure 4:- Some images related to plateaux investigations (lateritic soils). 

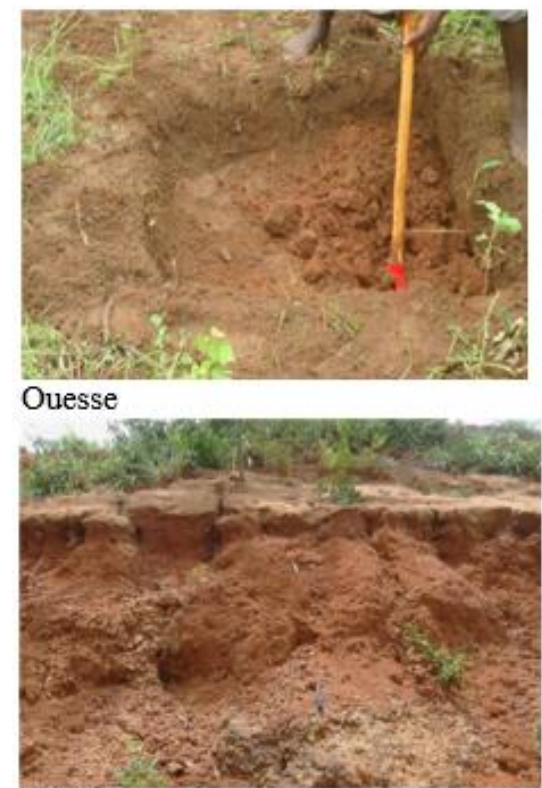

Nikki

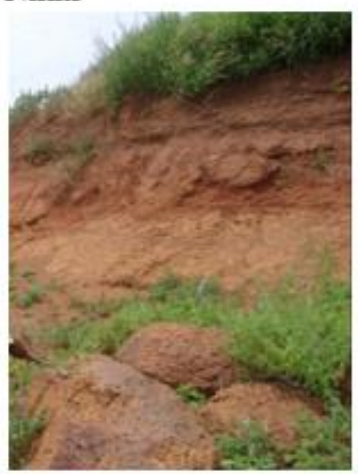

Banikoara

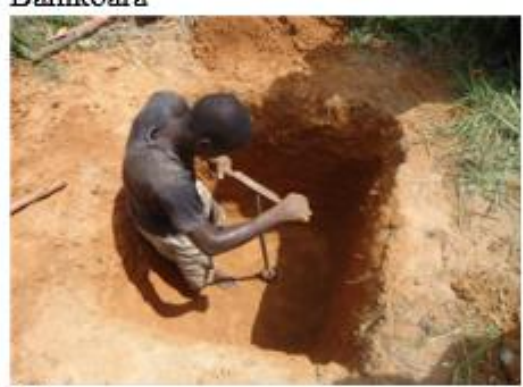

Djougou

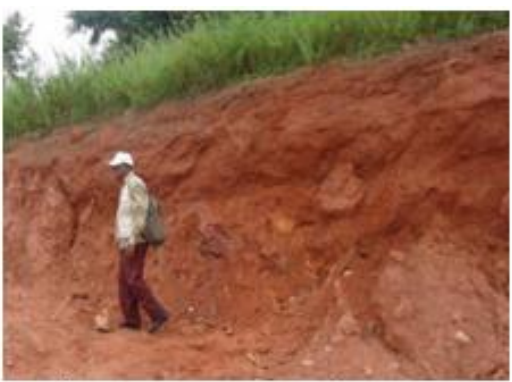

Parakou

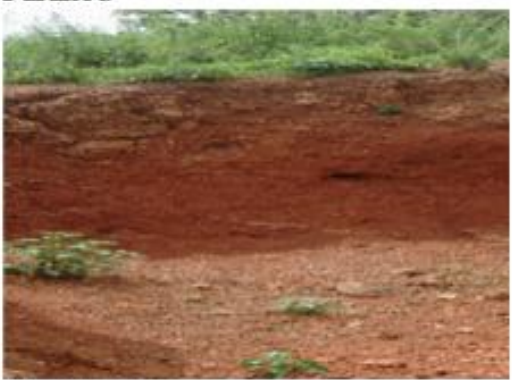

Kandi

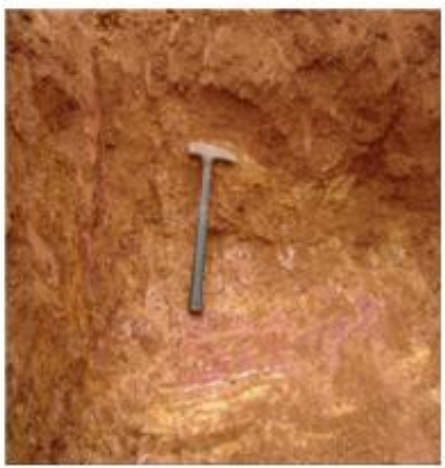

Bassila

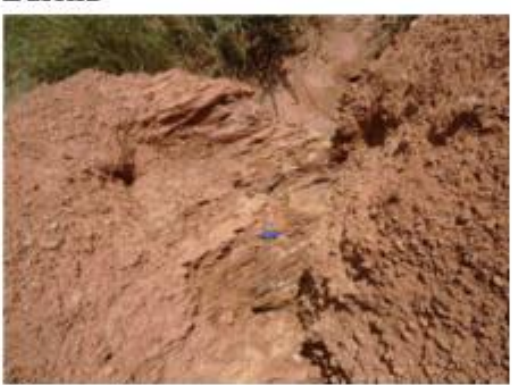

Tanguieta

Figure 5:- Some images of sites on the basement and Buem (Alterite)

There is a coherent appearance of the primeval lateritic soils (Fig 4). The color of these soils varies from one site to another. Nevertheless, it tends towards the brick red color. For alterites, their color is more or less hardened appearance is noted (Fig 5).

Fig 6 to Fig 10 show sections of clayey made on the plateaux. 


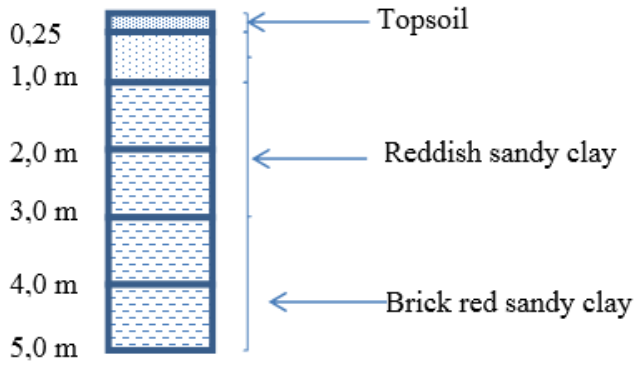

Figure 6 : Log of well dug in Adjarra - Allada - Ouidah - Toffo - Come - Bopa - Houeyogbe - Abomey - Lokossa - Dogbo - Aplahoue - Agbangnizoun - Zakpota
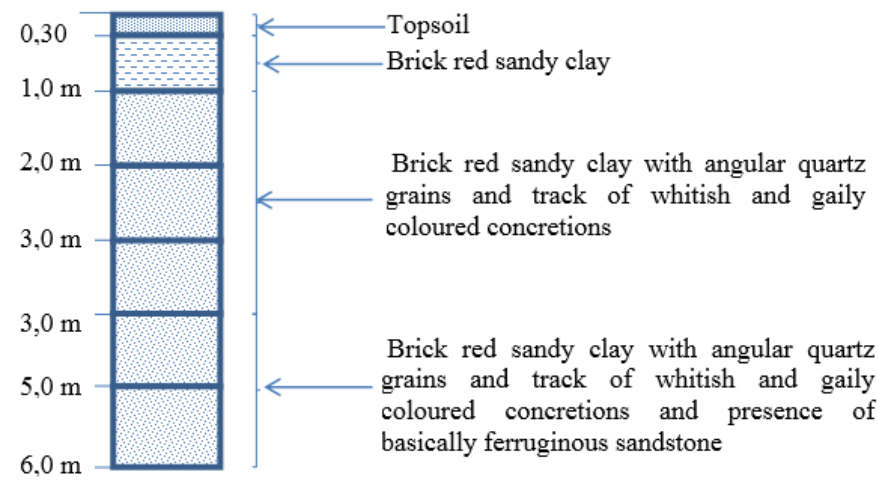

Figure 7 : Log of well dug in Sakete - Ikpinle

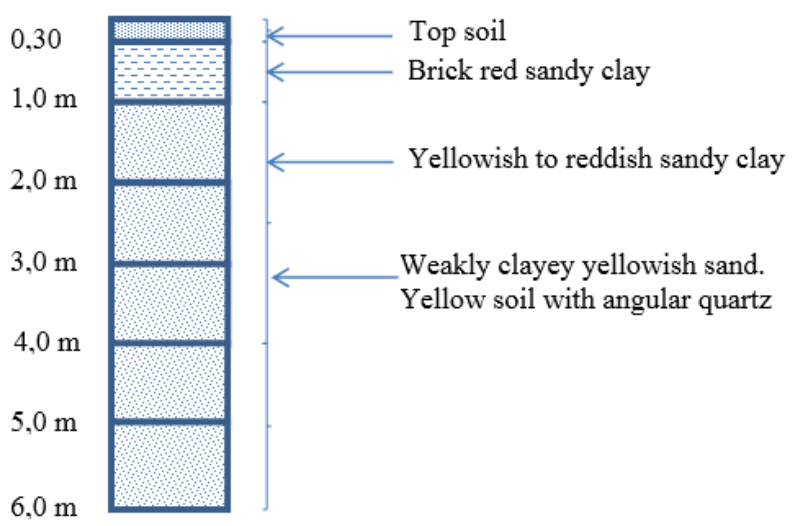

Figure 8 : Log of well dug in Abomey-Calavi

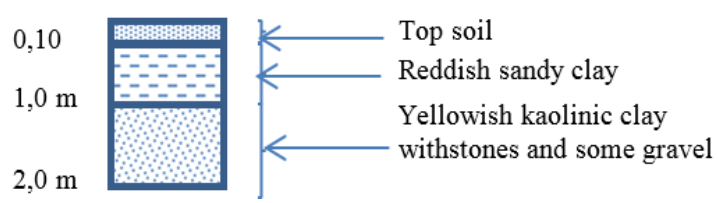

Figure 9: Log of well dug in Houeyogbe 


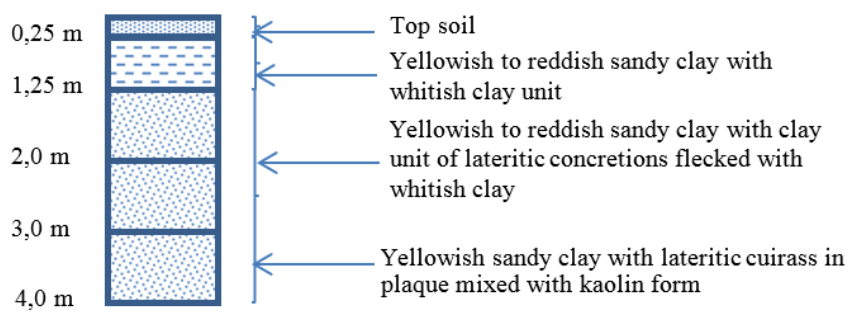

Figure 10: Log of well dug in Bohicon

The different logs and section of wells show homogeneity lateritic soils. Figure 6 shows this trend where topsoil, reddish sandy clay, and predominantly brick-red sandy clay are noted. Thus the same aspect was obtained for 13 sites out of 18: Adjarra - Allada - Ouidah - Toffo - Come - Bopa - Houeyogbe - Abomey - Lokossa - Dogbo Aplahoue - Agbangnizoun - Zakpota.

On the Sakete plateau (Fig 7), there are angular quartz grains and track of whitish and gaily coloured concretions with basically ferruginous sandstone. At Abomey-Calavi, slightly yellowish (silty) clayey sand has been identified (Fig 8); wells dug in Houeyogbe (Fig 9) and Bohicon (Fig 10) showed a yellowish kaolin clay.

The observation of plateaux located in the North of the Lama depression (Aplahoué, Abomey, Kétou, Zagnanado), between Lanta and Adjahonmè, along the Abomey-Azovè road showed that the brick red sand-clay formations, developed in fractured areas and on quartz sands with conglomerate levels and oblique starifications; they are alteration facies (IRB, 1985).

In Fig 11-a, the physical continuity of the gravelly horizons testifies the autochthonous character of the relics and the in-situ alteration of the clayey earth. Fig 11-b shows the clayey Earth within the sound rock in connection with particularly joint areas where fracture intensifies the pedogenetic mechanism.

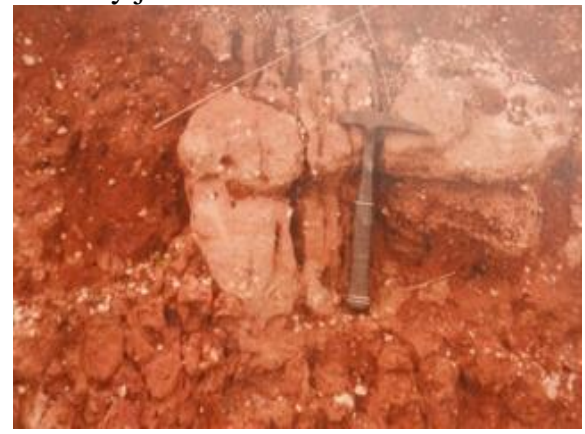

(a) Relics of healthy rock inside alteration materials Figure 11 : Healthy rock mix and alteration materials on the Abomey plateau (IRB, 1985)

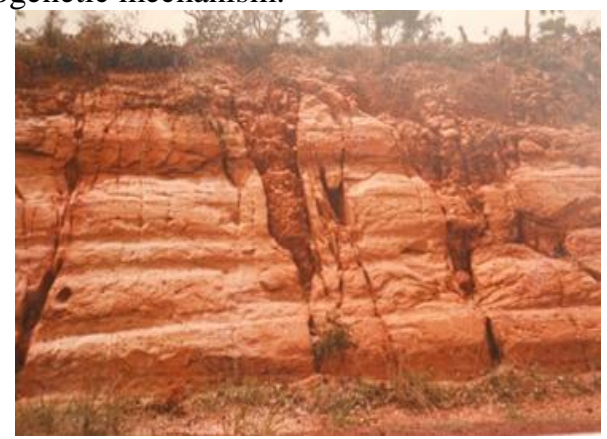

(b) Clayey soil within sound rock

The sections established for the basement and Buem alterites are shown from Fig 12 to Fig 17.

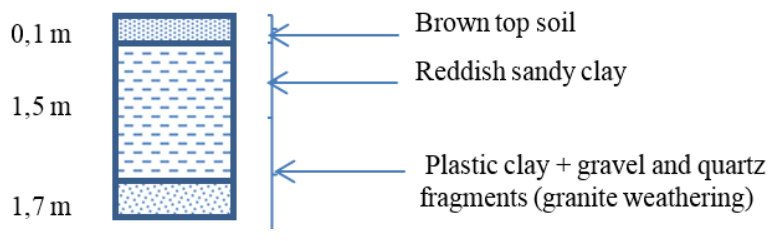

Figure 12: Log of well dug in Save 


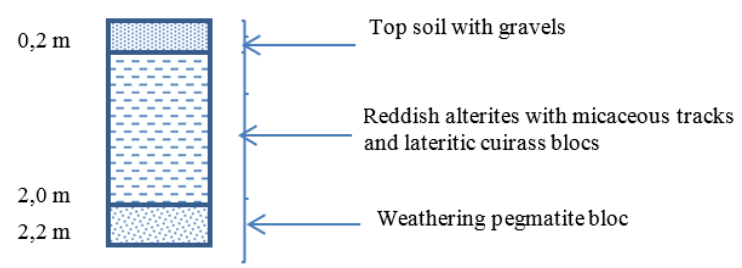

Figure 13: Log of well dug in Parakou- Tchaourou

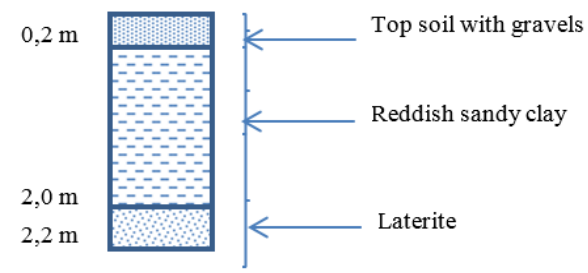

Figure 14:-Well sections in (Nikki - Kouandé - Tanguiéta)

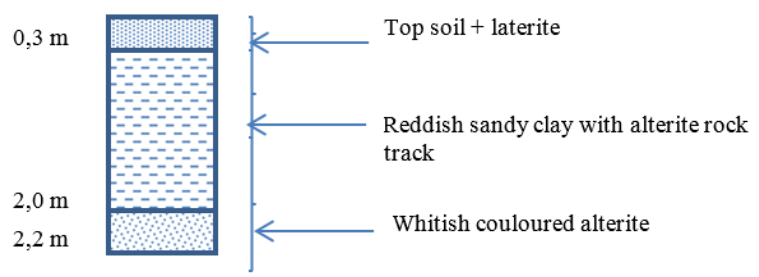

Figure 15:-Well sections Alibori (Kandi- Banikoara)

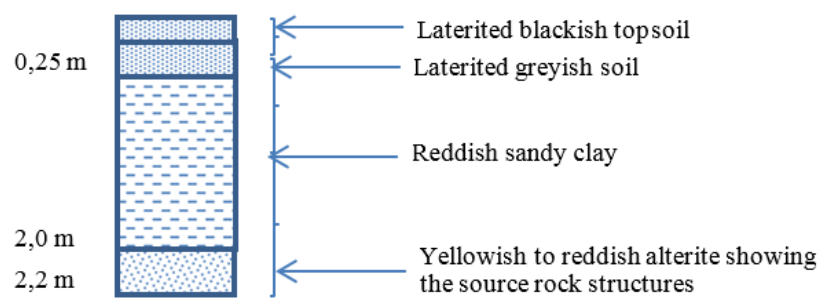

Figure 16:-Log of well dug in Bassila - Natitingou

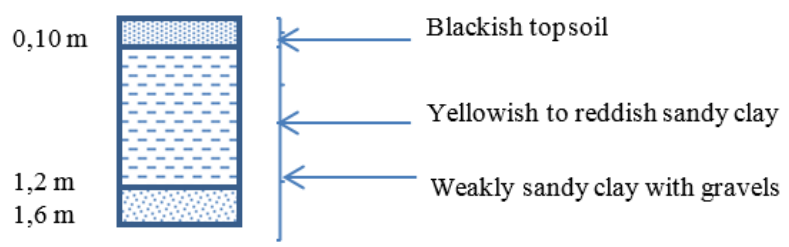

Figure 17:-Log of well dug in Djougou

There is a diversity with alterites. Laterite is mainly observed in Nikki - Kouande - Tanguieta (Fig 13); clay (Fig 11, Fig 16), weathering pegmatite block in Parakou and Tchaourou in the field of migmatites (Fig 12), whitish weathering alterite in Alibori (Fig 14) and reddish in Bassila - Natitingou (Fig 15).

Horizons A1, A2 and B are the one commonly sampled for alterite basement. Soils that are poor in clay and essentially leached (Fig 18). 


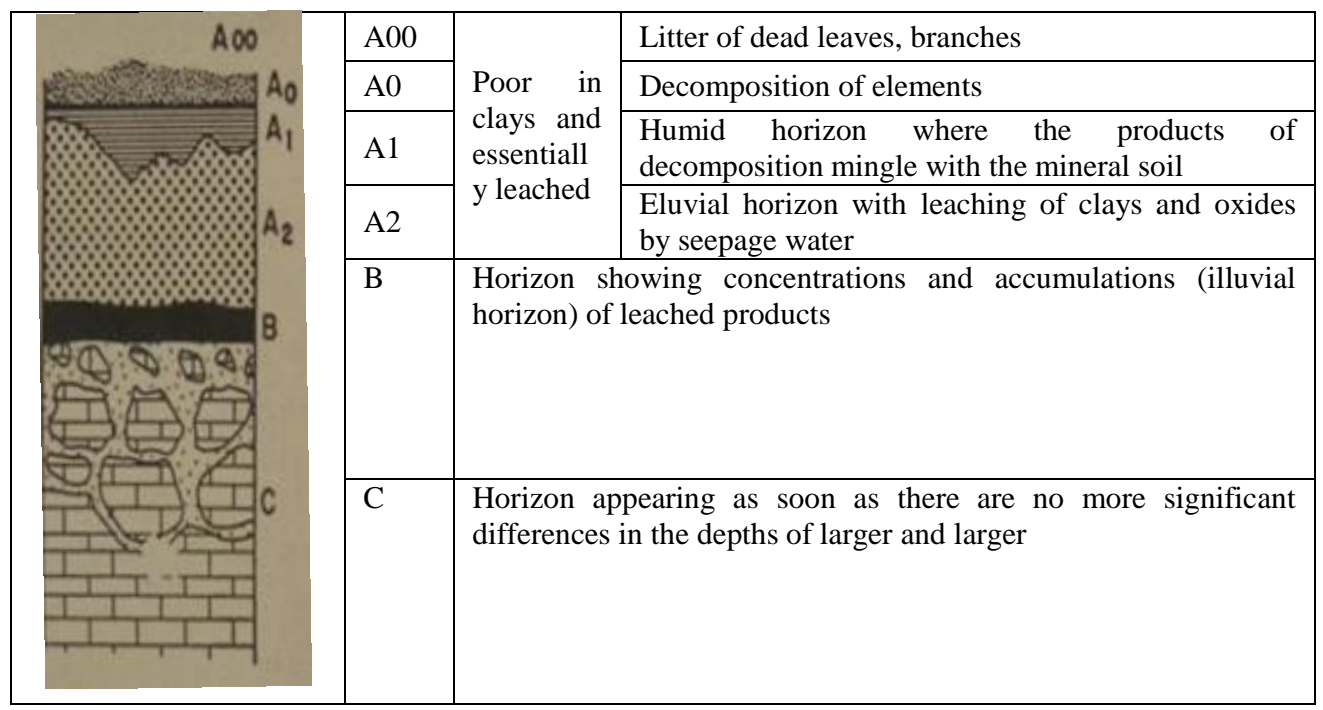

Figure 18: Soil's horizons (after Aubouin and al, 1975)

\section{Reserves evaluation}

According to the departments and the geological field, table 1 and table 2 present the explored clayey soil and laterite sites, their areas, the depth of the investigated wells, the minimum volumes and the geographical coordinates of the top of each site.

Table 1:-Prospected clayey soil Sites, their Geographic Coordinates and Reserves

\begin{tabular}{|c|c|c|c|c|c|c|c|c|c|c|}
\hline \multirow[b]{2}{*}{$\mathbf{N}^{\circ}$} & \multirow[b]{2}{*}{ Departments } & \multirow[b]{2}{*}{ Townships } & \multirow{2}{*}{$\begin{array}{l}\text { Geological } \\
\text { fields }\end{array}$} & \multirow{2}{*}{$\begin{array}{c}\text { Areas } \\
\left(\mathbf{m}^{2}\right)\end{array}$} & \multirow{2}{*}{$\begin{array}{c}\text { Thickn } \\
\text { ess } \\
\text { (m) }\end{array}$} & \multirow{2}{*}{$\begin{array}{c}\text { Reserves } \\
\left(\mathbf{m}^{3}\right)\end{array}$} & \multicolumn{4}{|c|}{ Geographic coordinates of the of the main sites'top } \\
\hline & & & & & & & $\mathbf{A}$ & \begin{tabular}{|c|}
$\mathbf{B}$ \\
\end{tabular} & $\mathbf{C}$ & D \\
\hline 1 & \multirow{3}{*}{ Atlantique } & $\begin{array}{l}\text { Abomey- } \\
\text { Calavi }\end{array}$ & \multirow{3}{*}{$\begin{array}{l}\text { Allada } \\
\text { Plateau }\end{array}$} & 48889 & 6 & 293332 & $\begin{array}{l}06^{\circ} 33^{\prime} 21.8^{\prime \prime} \mathrm{N} \\
02^{\circ} 19^{\prime} 28.2^{\prime \prime} \mathrm{E}\end{array}$ & \begin{tabular}{|l|}
$06^{\circ} 12^{\prime} 29.78^{\prime \prime} \mathrm{N}$ \\
$02^{\circ} 07^{\prime} 21.3{ }^{\prime \prime} \mathrm{E}$
\end{tabular} & $\begin{array}{l}06^{\circ} 33^{\prime} 20.5^{\prime \prime} \mathrm{N} \\
02^{\circ} 19^{\prime} 31.4^{\prime \prime} \mathrm{E}\end{array}$ & $\begin{array}{l}06^{\circ} 53^{\prime} 58.7^{\prime \prime} \mathrm{N} \\
02^{\circ} 19^{\prime} 31.1^{\prime \prime} \mathrm{E}\end{array}$ \\
\hline 2 & & Allada & & 7136 & 6 & 42819 & $\begin{array}{l}06^{\circ} 40^{\prime} 20.2^{\prime \prime}, \mathrm{N} \\
02^{\circ} 03^{\prime} 02.2^{\prime}{ }^{\prime} \mathrm{E}\end{array}$ & \begin{tabular}{|l|}
$06^{\circ} 40^{\prime} 20.2^{\prime}, \mathrm{N}$ \\
$02^{\circ} 08^{\prime} 03.1,{ }^{\prime}, \mathrm{E}$
\end{tabular} & $\begin{array}{l}06^{\circ} 40^{\prime} 18.6^{\prime \prime} \mathrm{N} \\
02^{\circ} 08^{\prime} 07.0^{\prime \prime} \mathrm{E}\end{array}$ & $\begin{array}{l}06^{\circ} 40^{\prime} 16.11^{\prime \prime} \mathrm{N} \\
02^{\circ} 08^{\prime} 06.2^{\prime \prime} \mathrm{E}\end{array}$ \\
\hline 3 & & Ouidah & & 1483885 & 6 & 8903312 & $\begin{array}{l}06^{\circ} 24^{\prime} 28.8^{\prime \prime} \mathrm{N} \\
02^{\circ} 10^{\prime} 00.8^{\prime \prime} \mathrm{E}\end{array}$ & $\begin{array}{l}06^{\circ} 24^{\prime} 27.6^{\prime \prime} \mathrm{N} \\
02^{\circ} 10^{\prime} 33.3, ' \mathrm{E}\end{array}$ & $\begin{array}{l}06^{\circ} 25^{\prime} 07.3^{\prime \prime} \mathrm{N} \\
02^{\circ} 10^{\prime} 33.7^{\prime \prime} \mathrm{E}\end{array}$ & $\begin{array}{l}06^{\circ} 25^{\prime} 07.9^{\prime \prime} \mathrm{N} \\
02^{\circ} 10^{\prime} 01.7^{\prime} \mathrm{E}\end{array}$ \\
\hline 4 & \multirow[t]{2}{*}{ Couffo } & Aplahoue & \multirow{3}{*}{$\begin{array}{l}\text { Aplahoue } \\
\text { Plateau }\end{array}$} & 6156 & 6 & 36935 & $\begin{array}{l}06^{\circ} 59^{\prime} 33.0^{\prime \prime} \mathrm{N} \\
01^{\circ} 43^{\prime} 52.8^{\prime \prime} \mathrm{E} \\
\end{array}$ & $\begin{array}{l}06^{\circ} 59^{\prime} 31.5^{\prime \prime} \mathrm{N} \\
01^{\circ} 43^{\prime} 55.4^{\prime \prime} \mathrm{E} \\
\end{array}$ & $\begin{array}{l}6^{\circ} 59^{\prime} 31.5^{\prime \prime} \mathrm{N} \\
01^{\circ} 43^{\prime} 51.7^{\prime \prime} \mathrm{E}\end{array}$ & $\begin{array}{l}06^{\circ} 59^{\prime} 29.6^{\prime \prime} \mathrm{N} \\
01^{\circ} 43^{\prime} 54.0^{\prime \prime} \mathrm{E} \\
\end{array}$ \\
\hline 5 & & Dogbo & & 12120 & 2,5 & 30299 & $\begin{array}{l}06^{\circ} 46^{\prime} 02.2^{\prime \prime} \mathrm{N} \\
01^{\circ} 46^{\prime} 12.9^{\prime}, \mathrm{E}\end{array}$ & $\begin{array}{l}06^{\circ} 46^{\prime} 02.2^{\prime \prime \prime} \mathrm{N} \\
01^{\circ} 46^{\prime} 15.4^{\prime \prime \prime} \mathrm{E}\end{array}$ & $\begin{array}{l}6^{\circ} 45^{\prime} 12.1^{\prime \prime} \mathrm{N} \\
01^{\circ} 46^{\prime} 12.1^{\prime \prime \prime} \mathrm{E}\end{array}$ & $\begin{array}{l}06^{\circ} 45^{\prime} 57.4^{\prime \prime} \mathrm{N} \\
01^{\circ} 46^{\prime} 14.0^{\prime \prime} \mathrm{E}\end{array}$ \\
\hline 6 & \multirow{4}{*}{ Mono } & Lokossa & & 3222 & 6 & 19330 & $\begin{array}{l}06^{\circ} 42^{\prime} 59.4^{\prime \prime} \mathrm{N} \\
01^{\circ} 47^{\prime} 48.7^{\prime \prime} \mathrm{E} \\
\end{array}$ & $\begin{array}{l}06^{\circ} 42^{\prime} 59.0^{\prime \prime} \mathrm{N} \\
01^{\circ} 47^{\prime} 49.6^{\prime \prime} \mathrm{E} \\
\end{array}$ & $\begin{array}{l}6^{\circ} 42^{\prime} 56.0^{\prime \prime} \mathrm{N} \\
01^{\circ} 47^{\prime} 47.5^{\prime \prime} \mathrm{E} \\
\end{array}$ & $\begin{array}{l}06^{\circ} 42^{\prime} 56.0^{\prime \prime} \mathrm{N} \\
01^{\circ} 47^{\prime} 47.5^{\prime \prime} \mathrm{E} \\
\end{array}$ \\
\hline 7 & & Bopa & \multirow{3}{*}{$\begin{array}{l}\text { Come } \\
\text { Plateau }\end{array}$} & 5239 & 6 & 31432 & $\begin{array}{l}06^{\circ} 30^{\prime} 37.8^{\prime \prime} \mathrm{N} \\
01^{\circ} 57^{\prime} 39.7^{\prime \prime} \mathrm{E}\end{array}$ & $\begin{array}{l}06^{\circ} 30^{\prime} 36.6^{\prime \prime} \mathrm{N} \\
01^{\circ} 57^{\prime} 41.0^{\prime \prime} \mathrm{E} \\
\end{array}$ & $\begin{array}{l}06^{\circ} 30^{\prime} 39.8^{\prime \prime} \mathrm{N} \\
01^{\circ} 57^{\prime} 43.0^{\prime \prime} \mathrm{E}\end{array}$ & $\begin{array}{l}06^{\circ} 30^{\prime} 40.4^{\prime \prime} \mathrm{N} \\
01^{\circ} 57^{\prime} 41.6^{\prime \prime} \mathrm{E}\end{array}$ \\
\hline 8 & & Come & & 53284 & 6 & 319704 & $\begin{array}{l}06^{\circ} 25^{\prime} 37.3^{\prime \prime \prime} \mathrm{N} \\
01^{\circ} 52^{\prime} 02.6^{\prime \prime} \mathrm{E}\end{array}$ & $\begin{array}{l}06^{\circ} 25^{\prime} 39.4^{\prime \prime} \mathrm{N} \\
01^{\circ} 52^{\prime} 08.9^{\prime \prime} \mathrm{E}\end{array}$ & $\begin{array}{l}06^{\circ} 25^{\prime} 48.8^{\prime \prime} \mathrm{N} \\
01^{\circ} 52^{\prime} 06.7^{\prime \prime} \mathrm{E}\end{array}$ & $\begin{array}{l}06^{\circ} 25^{\prime} 43.3^{\prime \prime} \mathrm{N} \\
01^{\circ} 52^{\prime} 00.4^{\prime \prime} \mathrm{E}\end{array}$ \\
\hline 9 & & Houeyogbe & & 2599 & 6 & 15592 & $\begin{array}{l}06^{\circ} 34,42.6^{\prime \prime} \mathrm{N} \\
01^{\circ} 51^{\prime} 13.0^{\prime \prime} \mathrm{E}\end{array}$ & $\begin{array}{l}6^{\circ} 34^{\prime} 43.0^{\prime \prime} \mathrm{N} \\
0^{\circ} 51^{\prime} 14.4^{\prime \prime} \mathrm{E}\end{array}$ & $\begin{array}{l}06^{\circ} 34^{\prime} 44.8^{\prime \prime} \mathrm{N} \\
01^{\circ} 51^{\prime} 13.9^{\prime \prime} \mathrm{E}\end{array}$ & $\begin{array}{l}06^{\circ} 34^{\prime} 44.3^{\prime \prime} \mathrm{N} \\
01^{\circ} 51^{\prime} 12.5^{\prime \prime} \mathrm{E}\end{array}$ \\
\hline 10 & Oueme & Adjarra & \multirow{3}{*}{$\begin{array}{l}\text { Sakete } \\
\text { Plateau }\end{array}$} & 6084 & 5 & 30421 & $\begin{array}{l}06^{\circ} 31^{\prime} 23.9^{\prime \prime} \mathrm{N} \\
02^{\circ} 41^{\prime} 11.6^{\prime \prime} \mathrm{E}\end{array}$ & $\begin{array}{l}06^{\circ} 31^{\prime} 22.4^{\prime \prime} \mathrm{N} \\
02^{\circ} 41^{\prime} 14.4^{\prime \prime} \mathrm{E} \\
\end{array}$ & $\begin{array}{l}6^{\circ} 31^{\prime} 22.1^{\prime \prime} \mathrm{N} \\
2^{\circ} 41^{\prime} 11.0^{\prime \prime} \mathrm{E} \\
\end{array}$ & $\begin{array}{l}6^{\circ} 31^{\prime} 21.6^{\prime \prime} \mathrm{N} \\
2^{\circ} 41^{\prime} 10.9^{\prime \prime} \mathrm{E} \\
\end{array}$ \\
\hline 11 & \multirow{3}{*}{ Plateau } & Adja-Ouere & & 24950 & 6 & 149702 & $\begin{array}{l}06^{\circ} 53^{\prime} 55.3^{\prime \prime \prime} \mathrm{N} \\
02^{\circ} 37^{\prime} 49.9^{\prime \prime} \mathrm{E}\end{array}$ & $\begin{array}{l}06^{\circ} 53^{\prime} 54.7^{\prime \prime} \mathrm{N} \\
02^{\circ} 37^{\prime} 58.2^{\prime}{ }^{\prime \prime} \mathrm{E} \\
\end{array}$ & $\begin{array}{l}06^{\circ} 53^{\prime} 51.8^{\prime \prime} \mathrm{N} \\
02^{\circ} 37^{\prime} 56.2^{\prime \prime} \mathrm{E} \\
\end{array}$ & $\begin{array}{l}06^{\circ} 53^{\prime} 58.7^{\prime \prime} \mathrm{N} \\
02^{\circ} 37^{\prime} 50.1^{\prime \prime} \mathrm{E} \\
\end{array}$ \\
\hline 12 & & Sakete & & 8843 & 6 & 53057 & $\begin{array}{l}6^{\circ} 43^{\prime} 11.6^{\prime \prime} \mathrm{N} \\
2^{\circ} 40^{\prime} 10.4^{\prime \prime} \mathrm{E}\end{array}$ & $\begin{array}{l}06^{\circ} 43^{\prime} 12.9^{\prime \prime} \mathrm{N} \\
02^{\circ} 40^{\prime} 08.7^{\prime}{ }^{\prime \prime} \mathrm{E}\end{array}$ & $\begin{array}{l}6^{\circ} 43^{\prime} 11.0^{\prime \prime} \mathrm{N} \\
2^{\circ} 40^{\prime} 07.4^{\prime \prime} \mathrm{E}\end{array}$ & $\begin{array}{l}6^{\circ} 43^{\prime} 07.3^{\prime \prime} \mathrm{N} \\
2^{\circ} 40^{\prime} 07.0^{\prime \prime} \mathrm{E}\end{array}$ \\
\hline 13 & & Ketou & $\begin{array}{l}\text { Ketou } \\
\text { Plateau }\end{array}$ & 11840 & 6 & 71041 & $\begin{array}{l}07^{\circ} 24^{\prime} 02.6^{\prime \prime} \mathrm{N} \\
02^{\circ} 37^{\prime} 39.0^{\prime \prime} \mathrm{E}\end{array}$ & $\begin{array}{l}07^{\circ} 24^{\prime} 03.4^{\prime \prime} \mathrm{N} \\
02^{\circ} 37^{\prime} 40.1^{\prime \prime} \mathrm{E}\end{array}$ & $\begin{array}{l}07^{\circ} 23^{\prime} 59.5^{\prime \prime} \mathrm{N} \\
02^{\circ} 37^{\prime} 36.6^{\prime \prime} \mathrm{E}\end{array}$ & $\begin{array}{l}07^{\circ} 23^{\prime} 56.7^{\prime \prime} \mathrm{N} \\
02^{\circ} 37^{\prime} 40.0^{\prime \prime} \mathrm{E}\end{array}$ \\
\hline 14 & \multirow{5}{*}{ Zou } & Zagnanado & $\begin{array}{l}\text { Zagnanado } \\
\text { Plateau }\end{array}$ & 12524 & 6 & 75145 & $\begin{array}{l}07^{\circ} 11^{\prime} 56.0^{\prime \prime} \mathrm{N} \\
02^{\circ} 22^{\prime} 57.9^{\prime \prime} \mathrm{E}\end{array}$ & $\begin{array}{l}07^{\circ} 11^{\prime} 53.8^{\prime \prime} \mathrm{N} \\
02^{\circ} 22^{\prime} 57.0^{\prime \prime} \mathrm{E} \\
\end{array}$ & $\begin{array}{l}07^{\circ} 11^{\prime} 51.6^{\prime \prime} \mathrm{N} \\
02^{\circ} 22^{\prime} 58.0^{\prime \prime} \mathrm{E}\end{array}$ & $\begin{array}{l}07^{\circ} 11^{\prime} 55.11^{\prime \prime} \mathrm{N} \\
02^{\circ} 23^{\prime} 01.8^{\prime \prime} \mathrm{E} \\
\end{array}$ \\
\hline 15 & & Abomey & \multirow{4}{*}{$\begin{array}{l}\text { Abomey } \\
\text { Plateau }\end{array}$} & 7679 & 2 & 15359 & $\begin{array}{l}07^{\circ} 11^{\prime} 52.3^{\prime \prime \prime} \mathrm{N} \\
02^{\circ} 58^{\prime} 51.1^{\prime \prime} \mathrm{E}\end{array}$ & $\begin{array}{l}07^{\circ} 11^{\prime} 55.0^{\prime \prime} \mathrm{N} \\
02^{\circ} 58^{\prime} 54.6^{\prime \prime} \mathrm{E} \\
\end{array}$ & $\begin{array}{l}07^{\circ} 11^{\prime} 50.0^{\prime \prime} \mathrm{N} \\
02^{\circ} 58^{\prime} 52.4^{\prime \prime} \mathrm{E}\end{array}$ & $\begin{array}{l}07^{\circ} 11^{\prime} 52.9^{\prime \prime} \mathrm{N} \\
02^{\circ} 58^{\prime} 54.6^{\prime \prime} \mathrm{E}\end{array}$ \\
\hline 16 & & Agbangnizoun & & 8059 & 6 & 48357 & $\begin{array}{l}07^{\circ} 07^{\prime} 42.7^{\prime \prime} \mathrm{N} \\
02^{\circ} 00^{\prime} 31.9^{\prime \prime} \mathrm{E} \\
\end{array}$ & $\begin{array}{l}07^{\circ} 07^{\prime} 40.9^{\prime \prime} \mathrm{N} \\
02^{\circ} 00^{\prime} 36.11^{\prime \prime} \mathrm{E} \\
\end{array}$ & $\begin{array}{l}07^{\circ} 07^{\prime} 39.8^{\prime \prime} \mathrm{N} \\
02^{\circ} 00^{\prime} 32.6^{\prime \prime} \mathrm{E} \\
\end{array}$ & $\begin{array}{l}07^{\circ} 07^{\prime} 39.1^{\prime \prime} \mathrm{N} \\
02^{\circ} 00^{\prime} 35.7^{\prime \prime} \mathrm{E} \\
\end{array}$ \\
\hline 17 & & Bohicon & & 15121 & 4 & 60486 & $\begin{array}{l}07^{\circ} 10^{\prime} 15.1^{\prime \prime} \mathrm{N} \\
02^{\circ} 06^{\prime} 29.33^{\prime \prime} \mathrm{E}\end{array}$ & $\begin{array}{l}7^{\circ} 10^{\prime} 19.3^{\prime \prime \prime} \mathrm{N} \\
02^{\circ} 06^{\prime} 31.9^{\prime \prime} \mathrm{E} \\
\end{array}$ & $\begin{array}{l}07^{\circ} 10^{\prime} 10.9^{\prime \prime} \mathrm{N} \\
02^{\circ} 06^{\prime} 32.1^{\prime \prime \prime} \mathrm{E}\end{array}$ & $\begin{array}{l}07^{\circ} 10^{\prime} 17.8^{\prime \prime} \mathrm{N} \\
02^{\circ} 06^{\prime} 34.1,{ }^{\prime \prime} \mathrm{E} \\
\end{array}$ \\
\hline 18 & & Za-Kpota & & 35770 & 6 & 214624 & $\begin{array}{l}07^{\circ} 13^{\prime} 58.9 .9^{\prime \prime} \mathrm{N} \\
02^{\circ} 12^{\prime} 42.3^{\prime}, \mathrm{E}\end{array}$ & \begin{tabular}{|l|}
$07^{\circ} 14^{\prime} 03.4^{\prime \prime} \mathrm{N}$ \\
$02^{\circ} 12^{\prime} 50.2^{\prime} \times \mathrm{N}$
\end{tabular} & $\begin{array}{l}7^{\circ} 13^{\prime} 55.2^{\prime \prime} \mathrm{N} \\
02^{\circ} 12^{\prime} 44.3^{\prime \prime} \mathrm{E}\end{array}$ & $\begin{array}{l}07^{\circ} 13^{\prime} 56.5^{\prime \prime} \mathrm{N} \\
02^{\circ} 12^{\prime} 50.1^{\prime \prime} \mathrm{E}\end{array}$ \\
\hline
\end{tabular}


Table 2:-Prospected alterite Sites, their Geographic Coordinates and Reserves

\begin{tabular}{|c|c|c|c|c|c|c|c|c|c|c|}
\hline \multirow{2}{*}{$\mathbf{N}^{\circ}$} & \multirow{2}{*}{$\begin{array}{l}\text { Depart } \\
\text { ments }\end{array}$} & \multirow{2}{*}{ Townships } & \multirow{2}{*}{$\begin{array}{l}\text { Geological } \\
\text { fields }\end{array}$} & \multirow{2}{*}{$\begin{array}{c}\text { Areas } \\
\left(\mathbf{m}^{2}\right)\end{array}$} & \multirow{2}{*}{$\begin{array}{l}\text { Thickness } \\
\text { (m) }\end{array}$} & \multirow{2}{*}{\begin{tabular}{|c|} 
Reserves \\
$\left(\mathbf{m}^{3}\right)$
\end{tabular}} & \multicolumn{4}{|c|}{ Geographic coordinates of the of the main sites'top } \\
\hline & & & & & & & $\mathbf{A}$ & B & C & \begin{tabular}{|l|} 
D \\
\end{tabular} \\
\hline 1 & & Banikoara & \begin{tabular}{|l} 
Gneiss, \\
Djougou- \\
Mekrou mica
\end{tabular} & 45466 & 3,2 & 145490 & $\begin{array}{l}11^{\circ} 18^{\prime} 45,2^{\prime \prime} \mathrm{N} \\
02^{\circ} 25^{\prime} 55,9^{\prime \prime} \mathrm{E}\end{array}$ & $\begin{array}{l}11^{\circ} 18^{\prime} 42,7^{\prime \prime} \mathrm{N} \\
02^{\circ} 26^{\prime} 02,0^{\prime \prime} \mathrm{E}\end{array}$ & $\begin{array}{l}11^{\circ} 18 ' 51,1^{\prime \prime} \mathrm{N} \\
02^{\circ} 26^{\prime} 03,2^{\prime \prime} \mathrm{E}\end{array}$ & $\begin{array}{l}11^{\circ} 18^{\prime} 54,0^{\prime \prime N} \\
02^{\circ} 25^{\prime} 59,7^{\prime \prime} \mathrm{E}\end{array}$ \\
\hline 2 & Alibori & Kandi & $\begin{array}{l}\text { Cam- Bro- } \\
\text { Silurians } \\
\text { Sandstone of } \\
\text { the Kandi } \\
\text { Sedimentary } \\
\text { Basin }\end{array}$ & 13994 & 2,2 & 30787 & $\begin{array}{l}11^{\circ} 12^{\prime} 13,9^{\prime \prime} \mathrm{N} \\
02^{\circ} 58^{\prime} 06,6^{\prime \prime} \mathrm{E}\end{array}$ & $\begin{array}{l}11^{\circ} 12^{\prime} 13,4^{\prime \prime} \mathrm{N} \\
02^{\circ} 58^{\prime} 05,3^{\prime \prime} \mathrm{E}\end{array}$ & $\begin{array}{l}11^{\circ} 12^{\prime} 07,0^{\prime \prime} \mathrm{N} \\
02^{\circ} 58^{\prime} 05,5^{\prime \prime} \mathrm{E}\end{array}$ & $\begin{array}{l}11^{\circ} 12^{\prime} 09,7^{\prime \prime N} \\
02^{\circ} 58^{\prime} 09,0^{\prime \prime} \mathrm{E}\end{array}$ \\
\hline 3 & Atacora & Kouande & \begin{tabular}{|l|} 
Donpago and \\
Kouande \\
Orthogneiss \\
with \\
muscovit \\
\end{tabular} & 50653 & 1,6 & 81044 & $\begin{array}{l}10^{\circ} 21^{\prime} 37,0^{\prime \prime} \mathrm{N} \\
01^{\circ} 41^{\prime} 25,9^{\prime \prime} \mathrm{E}\end{array}$ & $\begin{array}{l}10^{\circ} 21^{\prime} 11,0 \mathrm{~N} \\
01^{\circ} 41^{\prime} 30^{\prime \prime} \mathrm{E}\end{array}$ & $\begin{array}{l}10^{\circ} 21^{\prime} 14,2^{\prime \prime} \mathrm{N} \\
01^{\circ} 41^{\prime} 3,00^{\prime \prime} \mathrm{E}\end{array}$ & $\begin{array}{l}10^{\circ} 21^{\prime} 36,2^{\prime \prime N} \\
01^{\circ} 41^{\prime} 30,1^{\prime \prime} \mathrm{E}\end{array}$ \\
\hline 4 & Atacora & Natitingou & $\begin{array}{l}\text { Donpago and } \\
\text { Kouande } \\
\text { Orthogneiss } \\
\text { and quartzitic } \\
\text { Tectonic } \\
\text { Units of } \\
\text { Atacora }\end{array}$ & 32605 & 1,6 & 52167 & $\begin{array}{l}10^{\circ} 13^{\prime} 06,2^{\prime \prime} \mathrm{N} \\
01^{\circ} 24^{\prime} 02,3^{\prime \prime} \mathrm{E}\end{array}$ & $\begin{array}{l}10^{\circ} 13 \cdot 00,8^{\prime \prime N} \\
01^{\circ} 24^{\prime} 05,7^{\prime \prime E}\end{array}$ & $\begin{array}{l}10^{\circ} 13^{\prime} 04,6^{\prime \prime N} \\
01^{\circ} 24^{\prime} 10,3^{\prime \prime} \mathrm{E}\end{array}$ & $\begin{array}{l}10^{\circ} 13^{\prime} 09,7^{\prime \prime N} \\
01^{\circ} 24^{\prime} 04,9^{\prime \prime} \mathrm{E}\end{array}$ \\
\hline 5 & & Tanguieta & \begin{tabular}{|l|} 
Buem \\
sandstone and \\
silexites
\end{tabular} & 17745 & 1,8 & 31941 & $\begin{array}{l}10^{\circ} 45^{\prime} 32,0^{\prime \prime} \mathrm{N} \\
01^{\circ} 11^{\prime} 35,8^{\prime \prime} \mathrm{E}\end{array}$ & $\begin{array}{l}10^{\circ} 45^{\prime} 28,7 \mathrm{~N} \\
01^{\circ} 11^{\prime} 38,0 \mathrm{E}\end{array}$ & $\begin{array}{l}10^{\circ} 45^{\prime} 29,7^{\prime \prime} \mathrm{N} \\
01^{\circ} 11^{\prime} 41,2^{\prime \prime} \mathrm{E}\end{array}$ & $\begin{array}{l}10^{\circ} 45^{\prime} 34,6^{\prime \prime} \mathrm{N} \\
01^{\circ} 11^{\prime} 38,0^{\prime \prime} \mathrm{E}\end{array}$ \\
\hline 6 & & Nikki & $\begin{array}{l}\text { Migmatites } \\
\text { from Nikki- } \\
\text { Pèrèrè; gneiss } \\
\text { outcrop }\end{array}$ & 5553 & 2,2 & 12216 & $\begin{array}{l}09^{\circ} 55^{\prime} 35,8^{\prime \prime N} \\
03^{\circ} 04^{\prime} 54,6^{\prime \prime} \mathrm{E}\end{array}$ & $\begin{array}{l}09^{\circ} 55^{\prime} 32,6^{\prime \prime N} \\
03^{\circ} 04^{\prime} 24,5^{\prime \prime} \mathrm{E}\end{array}$ & $\begin{array}{l}09^{\circ} 55^{\prime} 32,9^{\prime \prime} \mathrm{N} \\
03^{\circ} 04^{\prime} 56,1^{\prime \prime} \mathrm{E}\end{array}$ & $\begin{array}{l}09^{\circ} 55^{\prime} 35,8^{\prime \prime} \mathrm{N} \\
03^{\circ} 04^{\prime} 56,5^{\prime \prime} \mathrm{E}\end{array}$ \\
\hline 7 & Borgou & Parakou & $\begin{array}{l}\text { Agramarou } \\
\text { migmatites; }\end{array}$ & 1442 & 2,7 & 3895 & \begin{tabular}{|l|}
$09^{\circ} 24^{\prime} 16,1^{\prime \prime N}$ \\
$02^{\circ} 37^{\prime} 14,7^{\prime \prime} \mathrm{E}$ \\
\end{tabular} & $\begin{array}{l}09^{\circ} 24^{\prime} 16,7^{\prime \prime N} \\
02^{\circ} 37^{\prime} 15,1 " \mathrm{E} \\
\end{array}$ & $\begin{array}{l}09^{\circ} 24^{\prime} 16,6^{\prime N} \mathrm{~N} \\
02^{\circ} 37^{\prime} 16,6^{\prime \prime} \mathrm{E} \\
\end{array}$ & \begin{tabular}{|l|}
$09^{\circ} 24^{\prime} 17,9^{\prime \prime N}$ \\
$02^{\circ} 37^{\prime} 16,4^{\prime \prime} \mathrm{E}$
\end{tabular} \\
\hline 8 & & Tchaourou & $\begin{array}{l}\text { presence of } \\
\text { large blocks } \\
\text { of lateritic } \\
\text { cuirasses }\end{array}$ & 38849 & 2,2 & 85468 & $\begin{array}{l}08^{\circ} 51^{\prime} 20,8^{\prime \prime N} \\
02^{\circ} 36^{\prime} 13,7^{\prime \prime E}\end{array}$ & $\begin{array}{l}08^{\circ} 51^{\prime} 14,1^{\prime \prime N} \\
02^{\circ} 36^{\prime} 16,1^{\prime \prime} \mathrm{E}\end{array}$ & $\begin{array}{l}08^{\circ} 51^{\prime} 17,7 " \mathrm{~N} \\
02^{\circ} 36^{\prime} 21 " 7 " \mathrm{E}\end{array}$ & $\begin{array}{l}08^{\circ} 51^{\prime} 22,4^{\prime \prime} \mathrm{N} \\
02^{\circ} 36^{\prime} 20,9^{\prime \prime} \mathrm{E}\end{array}$ \\
\hline 9 & & $\begin{array}{l}\text { Dassa- } \\
\text { Zoume }\end{array}$ & $\begin{array}{l}\text { Migmatic } \\
\text { Gneiss }\end{array}$ & 41804 & 1,3 & 54345 & $\begin{array}{l}07^{\circ} 46^{\prime} 08.0^{\prime \prime N} \\
02^{\circ} 20^{\prime} 10.5^{\prime \prime} \mathrm{E}\end{array}$ & $\begin{array}{l}07^{\circ} 46^{\prime} 14.2^{\prime \prime N} \\
02^{\circ} 20^{\prime} 15.1^{\prime \prime E}\end{array}$ & $\begin{array}{l}07^{\circ} 46^{\prime} 09.3^{\prime \prime} \mathrm{N} \\
02^{\circ} 20^{\prime} 20.5^{\prime \prime} \mathrm{E}\end{array}$ & $\begin{array}{l}07^{\circ} 46^{\prime} 05.5^{\prime \prime N} \\
02^{\circ} 20^{\prime} 16.0^{\prime \prime} \mathrm{E}\end{array}$ \\
\hline 10 & & Glazoue & $\begin{array}{l}\text { Biotite and } \\
\text { amphibole- } \\
\text { eyed gneiss }\end{array}$ & 7137 & 1,5 & 10706 & $\begin{array}{l}07^{\circ} 52^{\prime} 42.2^{\prime \prime} \mathrm{N} \\
02^{\circ} 12^{\prime} 29.2^{\prime \prime} \mathrm{E}\end{array}$ & $\begin{array}{l}07^{\circ} 52^{\prime} 41.4^{\prime \prime} \mathrm{N} \\
02^{\circ} 12^{\prime} 31.0^{\prime \prime} \mathrm{E}\end{array}$ & $\begin{array}{l}07^{\circ} 52^{\prime} 36.8^{\prime \prime} \mathrm{N} \\
02^{\circ} 12^{\prime} 30.0^{\prime \prime} \mathrm{E}\end{array}$ & $\begin{array}{l}07^{\circ} 52^{\prime} 39.7^{\prime \prime N} \\
02^{\circ} 12^{\prime} 28.3^{\prime \prime} \mathrm{E}\end{array}$ \\
\hline 11 & Collines & Ouesse & $\begin{array}{l}\text { Agramarou } \\
\text { migmatites; } \\
\text { granite } \\
\text { outcrop } \\
\end{array}$ & 19524 & 1,7 & 33191 & $\begin{array}{l}08^{\circ} 36^{\prime} 47,1^{\prime \prime N} \\
02^{\circ} 33^{\prime} 28,4^{\prime \prime} \mathrm{E}\end{array}$ & $\begin{array}{l}08^{\circ} 36^{\prime} 45,5^{\prime \prime} \mathrm{N} \\
02^{\circ} 33^{\prime} 25,2^{\prime \prime} \mathrm{E}\end{array}$ & $\begin{array}{l}08^{\circ} 36^{\prime} 43,1^{\prime \prime} \mathrm{N} \\
02^{\circ} 33^{\prime} 29,7^{\prime \prime} \mathrm{E}\end{array}$ & $\begin{array}{l}08^{\circ} 36^{\prime} 50,6^{\prime \prime} \mathrm{N} \\
02^{\circ} 33^{\prime} 31,9^{\prime \prime}\end{array}$ \\
\hline 12 & & Savalou & $\begin{array}{l}\text { Alkaline } \\
\text { pyroxene } \\
\text { gneiss }\end{array}$ & 9241 & 1,2 & 11089 & $\begin{array}{l}07^{\circ} 50^{\prime} 01.6^{\prime \prime} \mathrm{N} \\
01^{\circ} 59^{\prime} 53.9^{\prime \prime} \mathrm{E}\end{array}$ & $\begin{array}{l}07^{\circ} 50^{\prime} 02.4^{\prime \prime N} \\
01^{\circ} 59^{\prime} 55.1^{\prime \prime} \mathrm{E}\end{array}$ & $\begin{array}{l}07^{\circ} 50^{\prime} 03.11^{\prime \prime} \mathrm{N} \\
01^{\circ} 59^{\prime} 57.9^{\prime \prime} \mathrm{E}\end{array}$ & $\begin{array}{l}07^{\circ} 49^{\prime} 58.8^{\prime \prime} \mathrm{N} \\
01^{\circ} 59^{\prime} 58.5^{\prime \prime}\end{array}$ \\
\hline 13 & & Save & $\begin{array}{l}\text { Migmatic } \\
\text { Gneiss }\end{array}$ & 47640 & 1,2 & 57168 & $\begin{array}{l}08^{\circ} 11^{\prime} 41.7^{\prime \prime} \mathrm{N} \\
02^{\circ} 39^{\prime} 16.2^{\prime \prime} \mathrm{E}\end{array}$ & $\begin{array}{l}08^{\circ} 11^{\prime} 41.8^{\prime \prime} \mathrm{N} \\
02^{\circ} 39^{\prime} 24.6^{\prime \prime} \mathrm{E}\end{array}$ & $\begin{array}{l}08^{\circ} 11^{\prime} 36.2^{\prime \prime} \mathrm{N} \\
02^{\circ} 39^{\prime} 24.5^{\prime \prime} \mathrm{E}\end{array}$ & $\begin{array}{l}08^{\circ} 11^{\prime} 36.1^{\prime \prime} \mathrm{N} \\
02^{\circ} 39^{\prime} 16.2^{\prime \prime}\end{array}$ \\
\hline 14 & & Bassila & $\begin{array}{l}\text { Migmatites } \\
\text { and gneissic } \\
\text { formations }\end{array}$ & 25097 & 2,85 & 71528 & $\begin{array}{l}09^{\circ} 02^{\prime} 28,9^{\prime \prime} \mathrm{N} \\
01^{\circ} 39^{\prime} 10,1^{\prime \prime} \mathrm{E}\end{array}$ & $\begin{array}{l}09^{\circ} 02^{\prime} 24,2^{\prime \prime} \mathrm{N} \\
01^{\circ} 39^{\prime} 11,1^{\prime \prime} \mathrm{E}\end{array}$ & $\begin{array}{l}09^{\circ} 02^{\prime} 26,7^{\prime \prime} \mathrm{N} \\
01^{\circ} 39^{\prime} 16,7^{\prime \prime} \mathrm{E}\end{array}$ & $\begin{array}{l}09^{\circ} 02^{\prime} 30,4^{\prime \prime N} \\
01^{\circ} 39^{\prime} 16,7^{\prime \prime} \mathrm{E}\end{array}$ \\
\hline 15 & Donga & Djougou & $\begin{array}{l}\text { Gneiss, Mica } \\
\text { from } \\
\text { Djougou- } \\
\text { Mekrou }\end{array}$ & 27356 & 1,6 & 43770 & $\begin{array}{l}09^{\circ} 41^{\prime} 02,33^{\prime \prime N} \\
01^{\circ} 36^{\prime} 09,3^{\prime \prime} \mathrm{E}\end{array}$ & $\begin{array}{l}09^{\circ} 40^{\prime} 51,7^{\prime \prime N} \\
01^{\circ} 36^{\prime} 16,8^{\prime \prime} \mathrm{E}\end{array}$ & $\begin{array}{l}09^{\circ} 41,583^{\prime \prime N} \\
01^{\circ} 36^{\prime} 14,6^{\prime \prime} \mathrm{E}\end{array}$ & $\begin{array}{l}09^{\circ} 41^{\prime} 05,3^{\prime \prime N} \\
01^{\circ} 36^{\prime} 13,4^{\prime \prime} \mathrm{E}\end{array}$ \\
\hline
\end{tabular}

On the plateaux, the depths of the wells reach $6 \mathrm{~m}$. Those reserves are generally under estimated, for, numerous wells did not reach the level of the useful prospected layer. Thus on some sites as Adjarra, Ipinlè, Allada etc. the exploitation can go up to $10 \mathrm{~m}$ while the calculations of reserves took into account only a slice of $5 \mathrm{~m}$ of raw material. Generally speaking, the section power at the basement level is low (between 1.2 and $2.2 \mathrm{~m}$ ). This is offset by the existence of large exploitable land. The evaluated minimum reserves amounted to $10.410 .949 \mathrm{~m}^{3}$ lateritic soils on the plateaux of the coastal sedimentary basin (Atlantique, Oueme, Plateau, Mono, Couffo and Zou departments). The largest reserve is Ouidah $\left(8.903 .312 \mathrm{~m}^{3}\right)$ on the Allada plateau. The reserves amount to $724.806 \mathrm{~m}^{3}$ alterites in the 
basement, Buem and Kandi sedimentary basin areas (Collines, Borgou, Alibori, Atacora and Donga departments). The major sites are in Banikoara $\left(145.490 \mathrm{~m}^{3}\right)$ and Tchaourou $\left(85.468 \mathrm{~m}^{3}\right)$.

\section{Conclusion:-}

This study is an exploration of sites of raw materials used in the production of soil agglomerates in Benin Republic in order to make the local building materials accessible. Lateritic soils and alterite favorable sites have been identified in many departments over the country, on the plateaux of the sedimental coastal basin (clayey soil) and the crystalline basement, Buem and the sedimental basin of Kandi.

The well sections show a homogeneity as far as clay sandy formations named 'clayed soil' are concerned, with a brick red colour prevailing; whereas the alterite sites offer a variety. Moreover, clayey soil appears as primeval coherent soils while alterites are more or less hardened. In general, the section at the basement level varies from 1.2 to $2.2 \mathrm{~m}$ in comparison with the plateaux where the wells' depth can reach $5 \mathrm{~m}$. The confirmed clay reserves are evaluated at $10.410 .949 \mathrm{~m}^{3}$ and those of the laterite at $724.806 \mathrm{~m}^{3}$. Additional sites can also be explored. Thus, raw materials that can be used in the production of building local materials, such as agglomerate with stabilized soil exist and their exploitation should be welcome.

\section{Acknowledgement:-}

The Coordinator and the research team extend their sincere thanks to the West African Economic and Monetary Union (WAEMU) for funding the project as part of its Higher Education Support Program (PAES).

The team also thanks the mayors of the townships explored for the interest they showed to the project and for their assistance.

\section{References:-}

1. Affaton P. [1975 (1973)] -Étude géologique et structurale du Nord-Ouest Dahomey, du Nord-Togo et du Sud-Est de la Haute-Volta. Thèse $3^{\mathrm{e}}$ cycle Univ. Aix-Marseille III, Marseille, Fr. et Trav. Lab. Sci. Terre St-Jérôme, Marseille, Fr., sér. B, $n^{\circ} 10,201 p$.

2. Aubouin J., Brousse R., Lehman J-P.- Précis de géologie tome 1 Pétrologie, Dunod, Paris 1975

3. Houessou A., J. Lang (1978). Contribution à l'étude du « continental terminal » dans le Bénin méridional Sci. Géol., 31, 4, p.137-149.

4. Houessou A. et Lang J. (1979) - La terre de barre dans le Bénin méridional (Afrique de l'Ouest). Ass. Sénégal. Et. Quatern. Afr. Bull. liaison, 56, 49-58.

5. Igué A. M., Floquet A., Stahr K. (2005). Land use/ cover change and farming systems in central Benin. Bulletin de la Recherche Agronomique, $N^{\circ}$ 50, 23-37

6. Igué A.M., Houndagba C.J., Gaiser T., Stahr K. (2012). Accuracy of the Land Use/Cover classification in the Oueme Basin of Benin (West Africa). International Journal of AgriScience Vol. 2(2): 174-184, February 2012 www.inacj.com ISSN: 22286322@ International Academic Journals

7. I.R.B. (1982) - Etude de cartographie géologique et de prospection minière de reconnaissance au Nord du $11^{\text {ème }}$ parallèle. Rapport du projet FED.

8. I.R.B. (1985) - Etude de cartographie géologique et de prospection minière de reconnaissance au Sud du $9^{\text {ème }}$ parallèle. Rapport du projet FED. N'5100.11.13.015, p. 111

9. OBEMINES (1989-a) - Carte géologique à 1/200.000 feuilles Malanville, Kandi, Porga, Karimama et leur notice explicative : Mémoire $\mathrm{n}^{\circ} 2$. Office Béninois des Mines.

10. OBEMINES (1989-b) - Carte géologique à 1/200.000, feuilles Lokossa- Porto-Novo, Abomey, Zagnanado, Pira-Savè et leur notice explicative : Mémoire $\mathrm{n}^{\circ}$. Office Béninois des Mines.

11. OBEMINES (1995) - Carte géologique à 1/200.000, feuilles Sansanné-Mango, Natitingou, Bembéréké, Dunkassa et leurs notices explicatives : Mémoires ${ }^{\circ} 4$ et 5 . Office Béninois des Mines.

12. Slansky Maurice (1962). Contribution à l'étude géologique du bassin sédimentaire côtier du Dahomey et du Togo, Mémoires du Bureau de Recherches Géologiques et Minières.

13. Sorgho, B., Zerbo, L., Keita, I., Dembele, C., Plea, M., Sol, V., Gomina M., Blanchart, P. (2014). Strength and creep behavior of geomaterials for building with tannin addition. Materials and Structures 47:937-946. DOI 10.1617/s11527-0130104-7

14. Vedogbeton N. Oyédé, Olodo David, 1996. Rapport Général du Séminaire National sur la Problématique de l'exploitation du sable au Bénin. Les potentialités du Bénin en matériaux de construction alternatifs au sable marin au Bénin. Ministère de l'Environnement, de l'Habitat et de l'Urbanisme (MEHU), 20 p. 